\title{
Type 2 diabetes in a four-year-old child
}

\author{
Lindsay Sawatsky RD, Julie Halipchuk RN MN, Brandy Wicklow MD MSc
}

Cite as: CMAJ 2017 July 4;189:E888-90. doi: 10.1503/cmaj.170259

A four-year-old First Nations boy was referred by his family physician for evaluation of new-onset diabetes at a diabetes centre for children and adolescents in Winnipeg, Manitoba. Because of the high rates of type 2 diabetes in Manitoba First Nations children, ${ }^{1}$ the family physician had screened the boy for diabetes. At the time of screening, both the parents and child said that he had no symptoms, but on direct questioning, it became clear that he had been experiencing polyuria, polydipsia and nocturia for two months.

Our patient had been in the 97th percentile for weight at birth and was delivered vaginally following induction for polyhydramnios. His mother had had pregestational type 2 diabetes treated with insulin, and he had experienced persistent newborn hypoglycemia that required hospital admission. He was formula fed. His parents reported that he had minimal physical activity, a high intake of sugar-containing beverages, and poor sleep habits. Several of his family members had been given a diagnosis of type 2 diabetes: his mother (at the age of 15), his maternal grandparents, paternal grandfather, two aunts and two uncles. Our patient had two healthy siblings and there was no family history of type 1 diabetes or other autoimmune diseases.

On physical examination, he weighed $38.6 \mathrm{~kg}$ (> 97th percentile), was $107.6 \mathrm{~cm}$ tall (<50th percentile) and had a body mass index (BMI) $z$ score of 4.7 (BMI $33.3 \mathrm{~kg} / \mathrm{m}^{2}$; > 97th percentile). He had mild acanthosis nigricans. Fundoscopy, thyroid palpation, cardiac, respiratory and abdominal examinations were normal. He had a glycated hemoglobin $\left(\mathrm{HbA}_{1 \mathrm{c}}\right)$ level of $7.9 \%$ (normal 4\%-6\%), a fasting glucose of 9.1 (normal 3.3-6.0) $\mathrm{mmol} / \mathrm{L}$, an elevated cholesterol panel (total cholesterol 7.0 [normal < 4.6] mmol/L, triglycerides 1.7 [normal < 1.7] mmol/L, low-density lipoprotein 4.7 [normal < 3.0] $\mathrm{mmol} / \mathrm{L}$, apolipoprotein B 1.47 [normal < 1.2] g/L) and elevated liver function tests (aspartate aminotransferase 41 [normal 10-50 $\mathrm{U} / \mathrm{L}$ ], alanine aminotransferase 60 [normal 4-30 U/L]). Type 1 diabetes autoantibodies (GADA-A, IA-2, IA) were negative. As selfdeclared First Nations Oji-Cree heritage, he was tested for the hepatocyte nuclear factor-1 $\alpha$ (HNF-1 $\alpha$ ) polymorphism, which results in a decreased insulin secretion capacity; ${ }^{1}$ he harboured one copy of the G319S HNF-1 $\alpha$ risk allele. ${ }^{1}$ We diagnosed type 2 diabetes based on risk factors: obesity, acanthosis nigricans, First Nations heritage, family history of diabetes and exposure to hyperglycemia in utero. ${ }^{2}$

\section{KEY POINTS}

- The prevalence of type 2 diabetes in children is rising worldwide.

- People of Oji-Cree heritage are at increased risk for harbouring HNF-1 $\alpha$ polymorphism, which results in a decreased insulin secretion capacity.

- Type 2 diabetes must be considered in children with risk factors, despite age and lack of classic symptoms.

Because lifestyle changes have been shown to be successful in children with type 2 diabetes whose $\mathrm{HbA}_{1 \mathrm{c}}$ is less than $9 \%$ at diagnosis, ${ }^{3}$ we prescribed lifestyle modification, including daily physical activity and elimination of sugar-containing beverages. We reviewed signs of metabolic decompensation with his parents. In response to persistently elevated home capillary blood glucose testing, basal insulin was initiated three weeks postdiagnosis. The family discontinued this about four weeks later, unrelated to hypoglycemia or adverse effects of the therapy. One year after diagnosis, the patient's $\mathrm{HbA}_{1 \mathrm{c}}$ had risen to $9.7 \%$ and we initiated twice-daily premixed $30 / 70$ insulin. $\mathrm{His} \mathrm{HbA}_{1 \mathrm{c}}$ was $8.7 \%$ one month later and his parents reported that home blood glucose levels had normalized. We encouraged lifestyle modification in addition to insulin (Figure 1).

\section{Discussion}

The prevalence of type 2 diabetes in children is rising worldwide. Incidence rates vary, with estimates for the United States as high as 12.5 per 100000 children between the ages of 10 and 19 years in 2011-2012. ${ }^{4}$ The Canadian incidence is documented to be 1.54 per 100000 children younger than 18 years. ${ }^{5}$ In some regions, the incidence of type 2 diabetes in children now exceeds that of type 1 diabetes. ${ }^{1}$ Type 2 diabetes disproportionately affects Indigenous children. ${ }^{6}$ Increasing rates of type 2 diabetes in younger age groups may in part be a result of the increasing rates of children exposed to gestational and pregestational type 2 diabetes in utero, a risk factor for future glycemic dyscontrol. ${ }^{7}$ Breastfeeding has been shown to offer protection against type 2 diabetes in the offspring of mothers with gestational and pregestational diabetes. $^{7}$ 


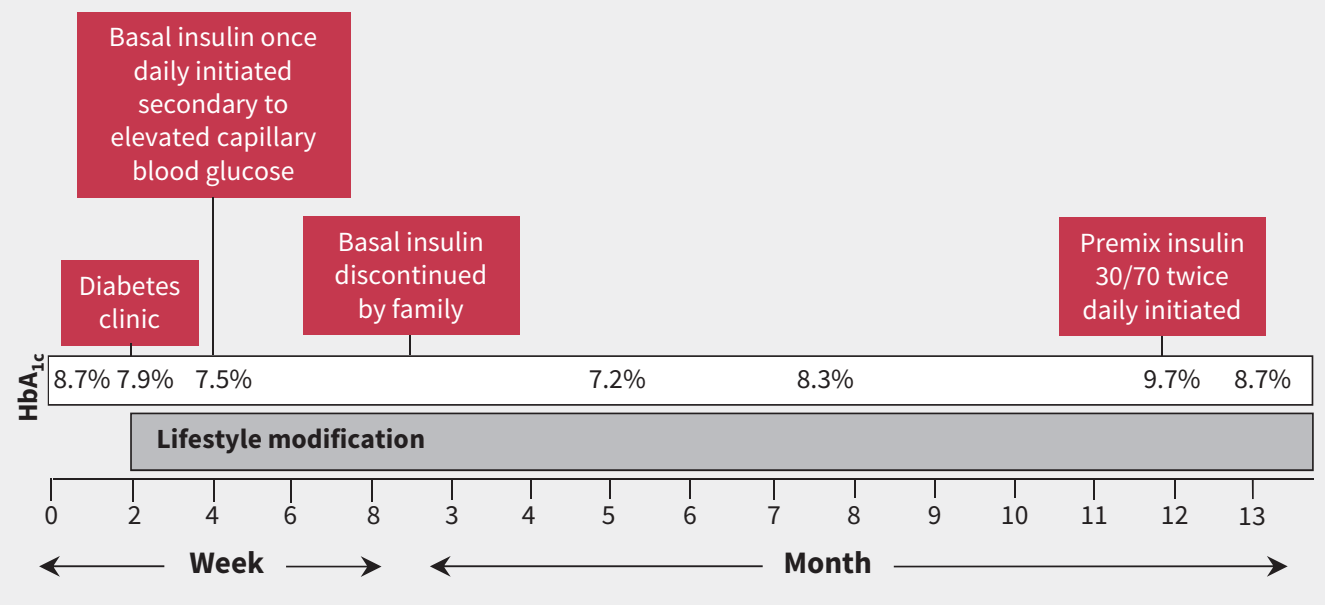

Figure 1: Timeline in weeks and months depicting clinical care of a boy diagnosed with type 2 diabetes at the age of four years. He was initially screened and given the diagnosis at week $0 . \mathrm{HbA}_{1 c}=$ glycated hemoglobin.

Childhood-onset type 2 diabetes is an emerging disorder most commonly diagnosed in midpuberty. ${ }^{1}$ However, type 2 diabetes in children younger than 10 years has been reported. The US SEARCH for Diabetes in Youth Study reported that children younger than 10 years comprised $3.6 \%$ of the total number of newly diagnosed cases of type 2 diabetes. ${ }^{8}$ One Canadian study found that $8 \%$ of children given a diagnosis of type 2 diabetes were younger than 10 years old. ${ }^{5}$ Type 2 diabetes is particularly prevalent in Manitoba, with a 20 -fold incidence compared with the rest of Canada. ${ }^{1}$ Incidence rates increased from 9.03/100 000 youth in 2006 to $20.55 / 100000$ youth in $2011 .{ }^{1}$ A review of the clinical database of the Diabetes Education Resource for Children and Adolescents in Winnipeg, Manitoba, from 2012 to 2016 found that $9.8 \%$ of 326 children given a type 2 diabetes diagnosis were younger than 10 (Appendix 1, available at www.cmaj.ca/ lookup/suppl/doi:10.1503/cmaj.170259/-/DC1).

Differentiation of type 1 and type 2 diabetes in children at the time of diagnosis can be difficult. It can take weeks to receive results of autoantibody testing and it therefore cannot be used at the time of diagnosis to classify diabetes. Although the presence of two or more diabetes autoantibodies (glutamic-aciddecarboxylase, islet antigen antibody and insulin antibodies) is used to classify type 1 diabetes, a 2015 study from the United Kingdom reported that $25.8 \%$ of children given a diagnosis of type 1 diabetes had no positive antibodies and $30 \%$ of children with a diagnosis of type 2 diabetes had at least one positive antibody. ${ }^{9}$ The presence of autoantibodies may suggest a more rapid decline in $\beta$-cell function and metabolic compromise. ${ }^{9}$ Our patient did not have any identified autoantibodies at diagnosis. A rapid decline in glycemic control in very young children suggests that the absence of antibodies does not correlate with a prolonged asymptomatic phase. In cases where classification of type of diabetes is unclear, it is important that glycemic control be closely monitored to avoid rapid decompensation resulting in diabetic ketoacidosis. Our patient's case raises questions about using age of onset as a distinguishing feature.

In Manitoba, we routinely screen for the HNF-1 $\alpha$ polymorphism in children with Oji-Cree heritage. Fifty-seven percent of the children described in Appendix 1 harbour at least one copy of the gene, and $25 \%$ of those children harbour two copies of the gene. A substantial portion of the attributable risk of diabetes results from a private variant in the HNF-1 $\alpha$ gene, in which a single nucleotide change results in the substitution of glycine for serine at codon 319 (HNF-1 $\alpha$ G319S). The presence of one or two copies of the "S-allele" increases risk for type 2 diabetes 10to 20 -fold compared with noncarriers. ${ }^{10}$ Impairment of insulin secretion in $\beta$ cells that express the G319S variant has been demonstrated. ${ }^{11}$

Type 2 diabetes must be considered in children with risk factors, despite age and lack of symptoms. Symptoms of hyperglycemia are not always apparent in children and cannot be relied on to identify those with type 2 diabetes, making it important to screen children at high risk regularly, even in the absence of symptoms. ${ }^{2}$ Our patient was wearing diapers, which may have affected his parents' assessment of his polyuria and nocturia. Diabetes Canada recommends screening for type 2 diabetes in prepubertal children who have three or more risk factors, and in pubertal youth who have two or more risk factors. ${ }^{2}$ In addition to the risk factors that our patient possessed, other risks include hypertension, dyslipidemia, nonalcoholic fatty liver disease and polycystic ovarian syndrome. ${ }^{2}$ In our centre, more than $50 \%$ of children younger than 10 years with type 2 diabetes had elevated cholesterol or liver enzymes.

\section{References}

1. Sellers EAC, Wicklow BA, Dean HJ. Clinical and demographic characteristics of type 2 diabetes in youth at diagnosis in Manitoba and Northwestern Ontario (2006-2001). Can J Diabetes 2012;36:114-8. 
2. Canadian Diabetes Association Clinical Practice Guidelines Expert Committee; Panagiotopoulos C, Riddell MC, Sellers EA. 2013 Clinical practice guidelines for the prevention and management of diabetes in Canada: type 2 diabetes in children \& adolescents. Can J Diabetes 2013;37:S163-7.

3. Wittmeier KD, Wicklow B, Sellers E, et al. Success with lifestyle monotherapy in youth with new-onset type 2 diabetes. Paediatr Child Health 2012;17:129-32.

4. Mayer-Davis EJ, Lawrence J, Dabelea D, et al. Incidence trends of type 1 and type 2 diabetes among youths; 2002-2012. N Engl J Med 2017;376:1419-29.

5. Amed S, Dean HJ, Panagiotopoulos C, et al. Type 2 diabetes, medicationinduced diabetes, and monogenic diabetes in Canadian children. Diabetes Care 2010;33:786-91.

6. Nadeau KJ, Anderson B, Berg EG, et al. Youth-onset type 2 diabetes consensus report: current status, challenges, and priorities. Diabetes Care 2016;39: 1635-42.

7. Halipchuk J, Temple B, Dart A, et al. Prenatal, obstetric and perinatal factors associated with the development of childhood-onset type 2 diabetes. Can J Diabetes 2017 June 2;pii:S1499-2671(16)30811-5. [Epub ahead of print] doi: 10.1016/j.jcjd.2017.04.003.

8. Writing Group for the SEARCH for Diabetes in Youth Study Group; Dabelea D, Bell RA, D'Agostino RB Jr., et al. Incidence of diabetes in youth in the United States. JAMA 2007;297:2716-24.

9. Perchard R, MacDonald D, Say J. Islet autoantibody status in a multi-ethnic UK clinic cohort of children presenting with diabetes. Arch Dis Child 2015;100: 348-52.

10. Hegele RA, Cao H, Harris S, et al. The hepatic nuclear factor-1alpha G319S variant is associated with early-onset type 2 diabetes in Canadian Oji-Cree. J Clin Endocrinol Metab 1999;84:1077-82.

11. Jonasson ME, Wicklow B, Sellers E, et al. Exploring the role of the HNF-1 $\alpha$ G319S polymorphism in $\beta$ cell failure and youth-onset type 2 diabetes: lessons from MODY and HNF-1 $\alpha$-deficient animal models. Biochem Cell Biol 2015;93: 487-94.

\section{Competing interests: None declared.}

This article has been peer reviewed.

The authors have obtained parental consent.

Affiliations: Health Sciences Centre Winnipeg (Sawatsky, Halipchuk); Department of Pediatrics and Child Health (Wicklow), Rady Faculty of Health Sciences, University of Manitoba; The Children's Hospital Research Institute of Manitoba (Wicklow), Winnipeg, Man.

Contributors: All authors contributed substantially to the conception and design of the manuscript. Lindsay Sawatsky acquired and analyzed the data, and drafted the initial manuscript. Julie Halipchuk and Brandy Wicklow reviewed and revised the manuscript for important intellectual content. All authors gave final approval of the version to be published and agree to act as guarantors of the work.

Correspondence to: Lindsay Sawatsky, Lsawatsky2@hsc.mb.ca

The section Cases presents brief case reports that convey clear, practical lessons. Preference is given to common presentations of important rare conditions, and important unusual presentations of common problems. Articles start with a case presentation (500 words maximum), and a discussion of the underlying condition follows (1000 words maximum). Visual elements (e.g., tables of the differential diagnosis, clinical features or diagnostic approach) are encouraged. Consent from patients for publication of their story is a necessity. See information for authors at www.cmaj.ca. 